TRABAJO EN PROGRESO

\title{
Uso de prototipos Lo-Fi en programación para niños
}

\author{
Nadia Selene Molina-Moreno, Maria Susana Avila- \\ Garcia, Marco Bianchetti, David Claudio-Gonzalez, \\ Marcelina Pantoja-Flores
}

Publicado: 21 Septiembre 2016

\begin{abstract}
Resumen
El uso de prototipos de baja fidelidad permite la obtención de las consideraciones de diseño para aplicaciones que permitan colaboración y participación de manera simultánea. El objetivo de este trabajo es analizar las interacciones sociales y con las herramientas de niños de entre 8 y 10 años durante el uso de prototipos de baja fidelidad para actividades donde se desarrolla la lógica de programación. En este trabajo se presentan resultados preliminares y se espera que los resultados obtenidos sean posteriormente utilizados para el desarrollo de herramientas interactivas y colaborativas en mesas multitáctiles, permitiendo la comunicación con el usuario, mostrando una interfaz fácil de manipular y operar.
\end{abstract}

Palabras clave: Pantalla Multitáctil; Programación para Niños; Prototipo Lo-Fi.

\section{Introducción}

En la actualidad los niños crecen a la par de la tecnología teniendo mayor acceso al uso de una computadora, tablet o teléfono celular. El avance de esta tecnología demanda que las nuevas generaciones estén capacitadas desde temprana edad para entender y aplicar conceptos de programación a diferentes niveles. Son varias las iniciativas e investigaciones, así como el desarrollo de herramientas de programación visual que permiten a los niños adquirir habilidades de programación de una manera fácil $[1,2]$. Las herramientas como Code.org, Scratch, Alice, GreenFood, BlueJ, y Karel el robot permiten a los niños adquirir habilidades de programación. Estas herramientas deben poseer características como la colaboración, dependencia, movilidad, portabilidad, entre algunas otras [3]. Para esto es necesario que el diseño de estas aplicaciones estén basados en las buenas prácticas de la Interacción Humano-Computadora, para que permita el uso adecuado de las mismas $[4,5]$. En este trabajo se propone el uso de prototipos de baja fidelidad (Lo-Fi) para que estudiar la manera en que los niños interactúan con las herramientas de programación

\footnotetext{
Molina-Moreno, NS., Avila-Garcia, MS., Bianchetti, M., ClaudioGonzalez, D., Pantoja-Flores, M.

Departamento de Estudios Multidisciplinarios, Universidad de Guanajuato

Av. Universidad S/N, Yuriria

Email: ns.molinamoreno@ugto.mx, susana.avila@ugto.mx,

mb@ugto.mx,d.claudiogonzalez@gmail.com,

m.pantoja.flores@ugto.mx
}

para que en un futuro estas interacciones sean traducidas, en la medida de lo posible, a un diseño en una aplicación para una mesa multitáctil, que además permita la organización y colaboración social gracias a su tamaño y características multi toque [6].

\section{Método}

Se realizó una revisión del estado del arte de las herramientas de programación visual y de los temas de programación adecuados para los niños [7]. Para este trabajo se seleccionó la herramienta Code.org (https://code.org/) debido a que cuenta con actividades guiadas de acuerdo a las edades de los niños y materiales para su desarrollo. Se creó un prototipo Lo-Fi, mostrado en Figura 1b, basado en un laberinto de pajaritos propuesto en Code.org en su curso número dos, como se muestra en la Figura 1a.

a)

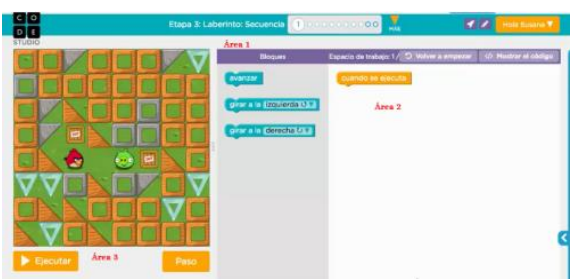

b)

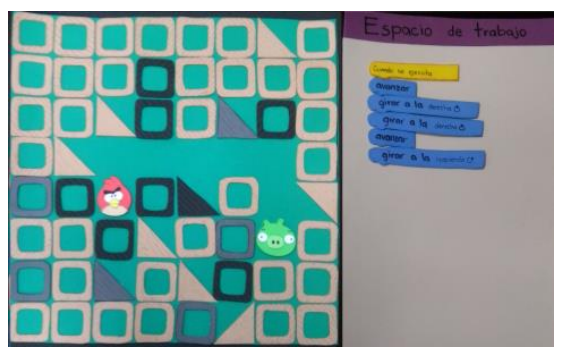

Figura 1. a) Actividad de la herramienta Code.org; b) Prototipo Lo-Fi

Esta aplicación en línea tiene tres áreas bien definidas: 1) caja de herramientas, 2) codificación (bloque color blanco donde se colocan las instrucciones), y 3) ejecución (laberinto). Estas áreas se muestran en la Figura 1a.

Para los experimentos, se llevaron a cabo tres sesiones con parejas de niños de entre 8 y 10 años. Se obtuvo el consentimiento de los padres para realizar la recolección de datos de los niños durante los experimentos y de su uso para fines de investigación. En las sesiones participaron tres aplicadores para las grabaciones y apoyo en la ejecución de los laberintos en prototipos Lo-Fi. 
Todas las sesiones fueron grabadas en video y se utilizó el software CamtasiaStudio para el procesamiento y análisis de los datos. Se utilizó una computadora y una cámara frente a la pareja de los niños para video grabar. En esta primera sección uno de los participantes fungía como el compilador y ejecutor de las instrucciones sobre el laberinto, moviendo el pajarito de acuerdo a la secuencia de instrucciones, definida por los niños, donde cada instrucción es representada por un bloque, la sesión se ilustra en la Figura 3. Como puede observarse, las herramientas de programación fueron colocadas a la derecha de los niños como área única, el área de codificación se colocó en un área común entre los niños y la parte de ejecución o laberinto cerca del ejecutor. En la Figura 3 se muestra a los niños definiendo la secuencia de instrucciones (a la izquierda), mientras que a la derecha se observa el aplicador que funge como compilador y ejecutor de las instrucciones.

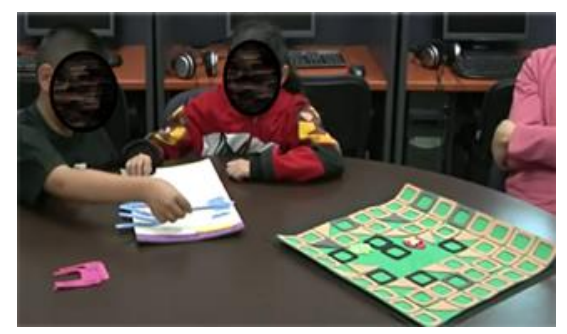

Figura 2. Sesión Lo-Fi

Los videos recolectados están siendo analizados de manera cualitativa, para lo cual se definieron rúbricas que permitieran capturar las interacciones entre los diferentes participantes del experimento así como de las interacciones [8] de los niños con el prototipo Lo-Fi. Se definieron claves para cada participante, aplicadores y niños, así como claves utilizadas para registrar la interacción entre los participantes y los elementos del prototipo Lo-Fi. Es importante mencionar que se incluyó también una clave que identifica la interacción de los niños con la cámara, esto con la finalidad de analizar la manera en que ésta pudo haber afectado su comportamiento y desempeño durante la realización de la actividad. La rúbrica contiene tiene 21 diferentes interacciones, cuyo objetivo es registrar las interacciones verbales, de gestos, y contacto con los elementos del prototipo.

De manera informal, se les cuestionó a los niños sobre su opinión de las actividades planteadas, de tal manera que sus comentarios puedan ser tomados en cuenta durante el diseño de las mismas [8]. Estos datos fueron registrados en una bitácora como anotaciones.

\section{Resultados preliminares y esperados}

Resultados preliminares muestran que el prototipo Lo-Fi fomenta la participación colaborativa y de manera simultánea, ya que pueden desarrollarse en un ambiente natural permitiendo el manejo total de la aplicación por más de un usuario. Un análisis de los diseños experimentales informará sobre recomendaciones para futuros experimentos en los que se tengan consideraciones como el uso de cámaras de video en las sesiones mientras que el análisis de los datos y la retroalimentación verbal de los niños serán importantes para la definición de recomendaciones sobre la distribución de las herramientas de programación, incluyendo las instrucciones, el área de codificación, y el área de ejecución (laberinto) en la mesa. La cantidad de interacciones de los participantes, el número de ocasiones que un participante toca un bloque o instrucción, si este es colocado en el área de codificación, si el bloque seleccionado está correcto, la manera en que los niños interactúan de manera simultánea con las herramientas y que herramientas son, será información que se espera derivar de la categorización de las interacciones basadas en las rúbricas antes mencionadas. De manera informal, se intenta recolectar recomendaciones realizadas por los niños para conocer más sobre sus preferencias. Todo esto resultará en una serie de recomendaciones de diseño de un prototipo funcional para una mesa multitáctil.

\section{Agradecimientos}

Los autores quieren agradecer a la Secretaría de Innovación, Ciencia y Educación Superior (SICES) por el apoyo brindado con el Proyecto "Análisis de Datos de Interacción en Actividades de Programación para Niños" de la Convocatoria Investigadores Jóvenes 2016. Nadia Selene Molina Moreno agradece al Consejo Nacional de Ciencia y Tecnología (CONACYT) por su apoyo con la Beca No. 649912.

\section{Referencias}

[1] Xiajian, C., W. Danli, and W. Hongan. Design and implementation of a graphical programming tool for children. In Computer Science and Automation Engineering (CSAE), 2011 IEEE International Conference on. 2011. IEEE.

[2] Sukamoto, H., et al. Programming education for primary school children using a textual programming language. In Frontiers in Education Conference (FIE), 2015. 326142015. IEEE. 2015. IEEE.

[3] Mohebbi, A., F. Asefi, and E.R. Tee. Common traits of graphical programming tools for children. in Engineering Education (ICEED), 2012 4th International Congress on. 2012. IEEE.

[4] Pane, J.F., B.A. Myers, and L.B. Miller. Using HCI techniques to design a more usable programming system. in Human Centric Computing Languages and Environments, 2002. Proceedings. IEEE 2002 Symposia on. 2002. IEEE.

[5] Fang, Z., W. Luo, and J. Xu. A structure for childrenoriented human computer interaction. In The Fourth International Workshop on Advanced Computational Intelligence. 201.

[6] Emma Mercier, G.V., Steve Higgins, Student interactions and the development of ideas inmulti-touch and paper-based collaborative mathematicalproblem solving. British Journal of Educational Technology, 2015: p. 2-14.

[7] Molina Moreno N. S. Selene, Avila Garcia, S., Claudio Gonzalez D., Bianchetti M., Pantoja Flores M., Análisis de aspectos colaborativos de herramientas de programación para niños. Congreso Internacional de Tecnologías y Computación., Dicienmbre 2015. 144: p. 349-360.

[8] Druin, A. (1999, May). Cooperative inquiry: developing new technologies for children with children. In Proceedings of the SIGCHI conference on Human Factors in Computing Systems (pp. 592-599). ACM. 